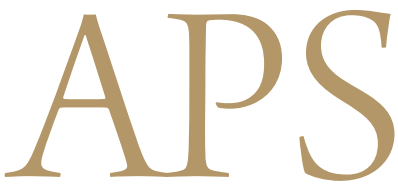

Archives of Plastic Surgery

\title{
Role of the Surgical Glove in Modified Vacuum-Assisted Wound Healing
}

\author{
Shankar Ram Hemmanur, Loka Vijayan Siddha \\ Department of Surgery, Kasturba Medical College, Manipal University, Mangalore, India
}

Vacuum-assisted wound healing has been proven to be more efficacious than conventional dressings. Vacuum dressing has been frequently modified given the restrictions in resources available. Here we present a modified method of vacuum dressing by using surgical or gynaecological gloves for lower and upper limb wounds. Vacuum dressing was applied with parts of a surgical or gynaecological glove and Opsite with T-tailing of the suction outlet. Vacuum-assisted wound healing using the surgical gloves showed relatively good wound healing in the amputation stump, finger, arm, and leg in the cases studied.

Keywords Surgical gloves / Vacuum / Occlusive dressing

Correspondence: Loka Vijayan Siddha Department of General Surgery, Kasturba Medical College,

Manipal University, Mangalore, India Tel: +9901832864

Fax +994662267206

E-mail: lokavijayan@gmail.com

We thank Prof Dr. Thangam Varghese, Prof Dr. Anand Kini, Prof Dr. Harish Rao, Department of General Surgery, KMC, Mangalore for their support and guidance.

No potential conflict of interest relevant to this article was reported.

\section{INTRODUCTION}

Vacuum-assisted wound healing $[1,2]$ has been proven to be more efficacious than conventional dressings. The surgical glove is cheap, easily available, sterile, elastic, and effectively seals once snugly fit. It is malleable such that it can be applied to a part of the body with a multifaceted contour, and it takes its shape under a vacuum while maintaining it. On this basis an attempt has been made to use a surgical or gynaecological glove as a modified form of vacuum-assisted wound healing with the help of Opsite and a T-tail configuration of the suction tubing outlet [3].

\section{IDEA}

Under aseptic conditions, the wound was thoroughly debrided and cleaned of slough and necrotic tissue. The wound base was covered with a sterile sponge placed in two layers. An infant feeding tube or suction catheter or Ryle's tube is placed in between the two layers. The sponge is held in place over the wound by applying thin Opsite strips like Steri-Strips. A sterile surgical or gynaecological glove of appropriate size is taken and inserted over the foot, hand, or amputation stump (Fig. 1). The suction tube is brought out from the edge of the glove. A $15 \mathrm{~cm} \times 28 \mathrm{~cm}$ piece of Opsite is taken and cut in half along its length and applied covering entire margin of the stretched glove circumferentially. The edges can also be fixed with skin staples. The Opsite should not be put too tight in order to prevent skin excoriation and peripheral edema.

1) Surgical gloves are available in various sizes. Gynaecological gloves of $71 / 2$ and $81 / 2$ sizes can be applied for lengthy wounds.

2) The arms of the gynaecological gloves can be cut and applied in an overlapping manner over the ends, in a tele-

Copyright $(\odot 2013$ The Korean Society of Plastic and Reconstructive Surgeons

This is an Open Access article distributed under the terms of the Creative Commons Attribution Non-Commercial License (http://creativecommons.org/

licenses/by-nc/3.0/) which permits unrestricted non-commercial use, distribution, and reproduction in any medium, provided the original work is properly cited 
scopic fashion so that larger lengths can be constructed, and there is very minimal leakage through the overlapping ends of the gloves. This overlapping holds a vacuum and prevents leakage when a continuous suction is applied and does not require any adhesive tape or Opsite for sealing.

3) A circumferential Opsite occlusion seal with T-tail configuration [3] of the suction tube outlet is applied only at the end (s) of the wound. Once suction is applied, the shrinkage of the sponge beneath the glove can be appreciated over the glove. Surgical glove fits well to the hand and also the fingers of the surgical glove can be cut and applied for isolated finger injuries.

4) A gynaecological glove can be cut obliquely to obtain a

\section{Fig. 1. Measurements and oblique slit mark on gloves}

Various measurements of the surgical and gynaecological gloves have been marked. The gloves can be cut and applied according to the length and size of the wound. An oblique cut (red line) can be made at an appropriate angle to obtain a wider opening in the arms of the gynaecological gloves and can be applied to areas like amputation stump ulcers.

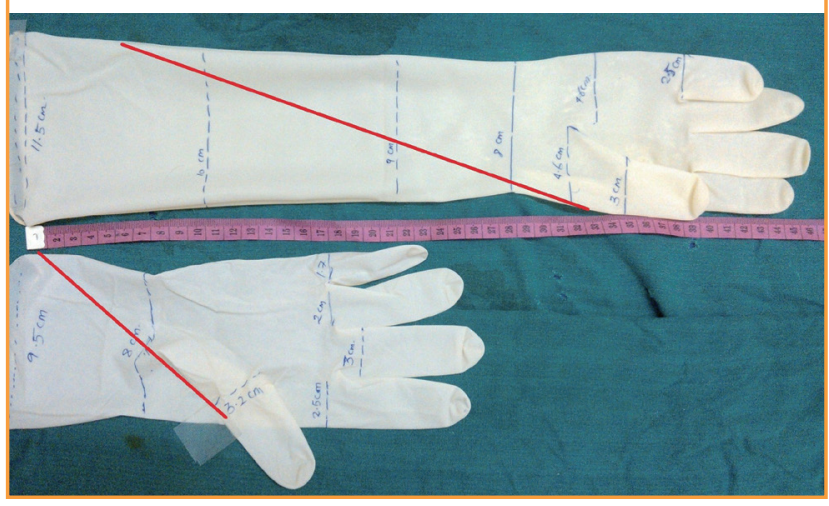

\section{Fig. 2. Intussuscepting glove arms with end Opsite cuffs}

For lengthy wounds, multiple gynaecological gloves can be cut and applied in a sequential overlapping manner.

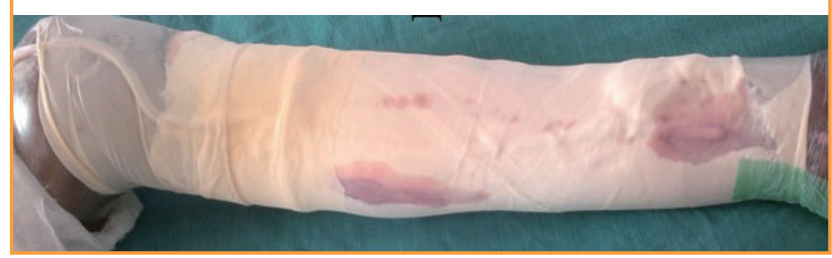

\section{Fig. 3. Leg wound healing 12 days after dressing}

A necrotising fasciitis wound on the left leg was debrided and the glove vacuum was applied for 6 dressings. The wound is pictured here after being allowed to heal for 12 days. Later skin grafting was performed.

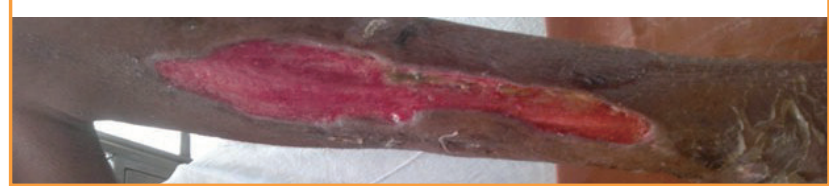

greater circumference, which is similar to the cheatle slit in right hemicolectomy.

5) An intermittent vacuum is applied for 20 minutes of every hour at a negative pressure of $125 \mathrm{~mm} \mathrm{Hg}$.

To reduce maceration over the plantar region, the surface is covered by collagen, glycerine magnesium sulphate, or zinc oxide. A 12 -hour to 24-hour gap between changing vacuum dressings has been observed to decrease maceration.

\section{DISCUSSION}

In attempts to use vacuum-assisted wound healing at the extremities, covering most of the wound area by an inexpensive surgical or gynaecological glove rather than Opsite significantly reduces the cost of each dressing. More sterile surgical and gynaecological gloves are readily available for this purpose. Opsite is used judiciously like a bottle cork to create a seal and cuff the ends of the glove vacuum unit. The surgical or gynaecological glove can be applied to the arms, legs, digits, and foot but is difficult for the adult thighs. The presence of external (Figs. 2-6) fixators would stretch the glove too much. Designing or altering sterile gloves or latex stockings for the purpose of vacuum dressing according to the need and contour of the site would further simplify the dressing In addition, the time required for each

Fig. 4. Glove vacuum covering the dorsum of the foot and digits

A glove vacuum was applied to a second degree burn wound on the right dorsum of the foot and the wound healed successfully.

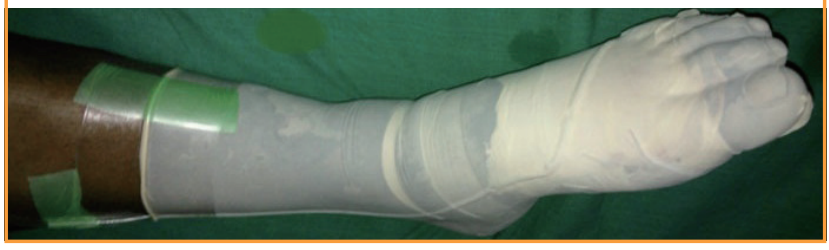

Fig. 5. Glove vacuum applied to arm

A necrotising fasciitis wound over the right arm was debrided and a glove vacuum was applied for 5 dressings and later skin grafting was performed.

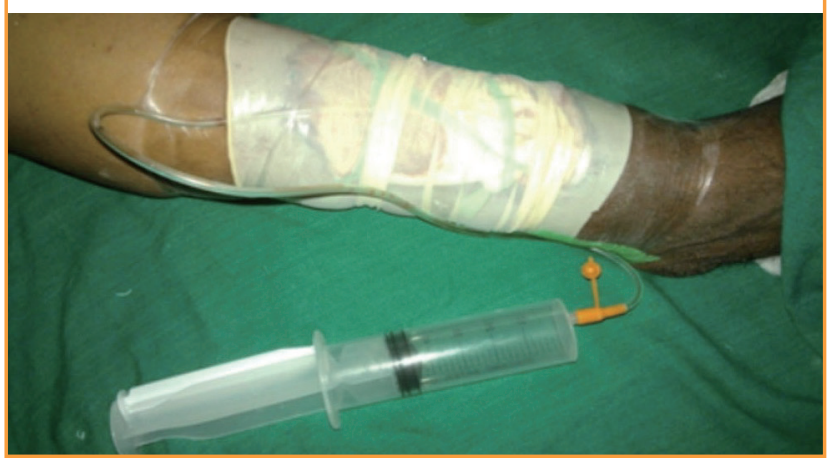




\section{Fig. 6. Amputation stump necrosis}

(A) A diabetic gangrenous right lower limb for which above-knee amputation was done followed by stump necrosis wound debridement. (B) Glove vacuum over above-knee amputation stump. (C) Glove vacuum was applied for 15 dressings and exposed bone was completely covered by granulation tissue. Later skin grafting was performed.
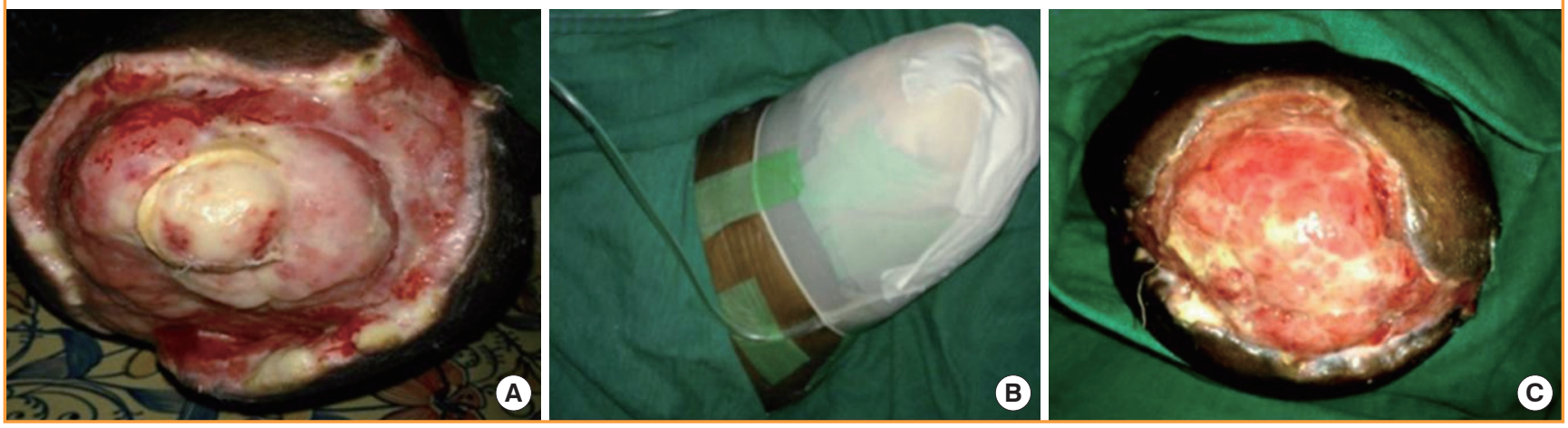

vacuum dressing application can be reduced. The efficacy of this modified surgical glove vacuum dressing should be compared to conventional vacuum dressings in further studies. In this series, the results were very effective.

\section{REFERENCES}

1. KCI. V.A.C. therapy for wounds [Internet]. Bangalore: KCI Medical India Pvt. Ltd.; c2013 [cited 2013 May 16]. Avail- able form: http://www.kcil.com/KCI1/vactherapy.

2. Satish Rao B, Shenoy H, Ravikrishnan J, et al. A comparative study of the efficacy of topical negative pressure moist dressings and conventional moist dressings in chronic wounds Indian J Plast Surg 2007;40:133-40.

3. Ram S, Vijayan SK, Kini A. T tail configuration of opsite on suction tubing outlet for modified vacuum assisted closure. Internet J Plast Surg 2012;8. http://dx.doi.org/10.5580/2cb6. 